\title{
Silicon Nanowire pH Sensors Fabricated Using Conventional CMOS Technologies
}

\author{
Zhida Zhao ${ }^{1}$, Kun Zhou ${ }^{1}$, Liyang Pan $^{1}$, Zheyao Wang ${ }^{1}$ \\ Institute of Microelectronics, Tsinghua University, Beijing 100084, China \\ z.wang@tsinghua.edu.cn
}

\begin{abstract}
:
This paper reports a fabrication method and an integration scheme for silicon nanowire (SNW) $\mathrm{pH}$ sensors. By using sidewall techniques, SNW with feature size down to nanometer regime can be obtained using conventional CMOS technology. The SNWs are operated in a FET configuration as a $\mathrm{pH}$ sensor, and sensitivity as high as $54.5 \mathrm{mV} / \mathrm{pH}$ for $\mathrm{pH}$ values from 1 to 12 is achieved.
\end{abstract}

Key words: silicon nanowire, CMOS, $\mathrm{pH}$ sensor, sidewall mask.

\section{Introduction}

Measurement of $\mathrm{pH}$ values are essential to chemical or biological applications such as environment and water quality monitoring, blood $\mathrm{pH}$ measurements, scientific research, DNA sequencing, and as many others [1]. A silicon nanowire (SNW) can measure $\mathrm{pH}$ values by operated in an ion-sensitive field effect transistor (ISFET). The carrier density of SNW channel is modulated through an electrostatic potential applied to the solution at the gate position. Because of the large surface-tovolume ratio of SNWs, high sensitivity has been achieved for SWN ISFETs in measurement of either $\mathrm{pH}$ or other biochemical targets [2].

One challenge for development of SNW sensors is that fabrication depends on patterning of tens or few hundreds of nano meter feature size. E-beam lithography is the most straightforward technique to fabricate nanowires [3], but the throughput and the cost are unsatisfactory. Advanced lithography such as extreme ultraviolet (EUV) is able to generate nanometer patterns with high throughput [4], but it is costly, inflexible, and hard for available. To solve this problem, this paper presents an approach to fabricate nanowires by using sidewall mask technology.

\section{Sensor Fabrication}

Fig. 1 shows the processes for fabrication of SNWs using sidewall mask technique. It starts with a silicon-on-insulator (SOI) wafer, which consists of a silicon device layer, a buried $\mathrm{SiO}_{2}$ layer (BOX), and a substrate, see Fig. 1(a). A $\mathrm{SiO}_{2}$ layer is deposited on the SOl wafer using plasma-enhanced chemical vapor deposition (PECVD), as shown in Fig. 1(b). The $\mathrm{SiO}_{2}$ layer is patterned and etched using reactive ion etching (RIE), see Fig. 1(c).

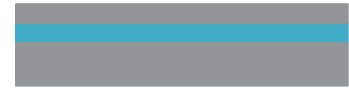

(a) SOI

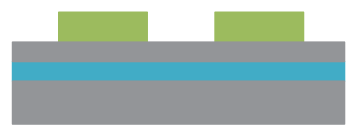

(c) Photolithogragh Plasma etching

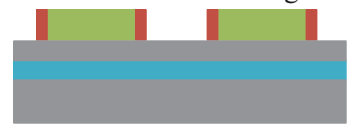

(e) Anisotropic Plasma

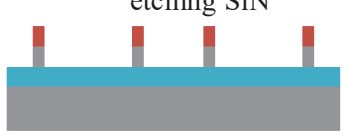

(g) $\begin{gathered}\text { Anisotropic Plasm } \\ \text { etching } \mathrm{Si}\end{gathered}$

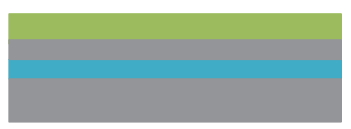

(b) $\mathrm{PECVD} \mathrm{SiO}_{2}$

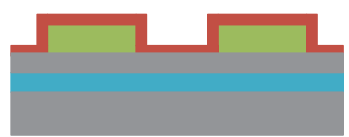

(d) PECVD SiN

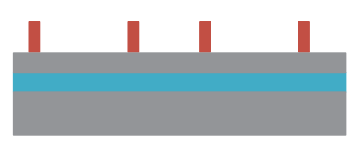

(f) Wet etching $\mathrm{SiO}_{2}$ with HF

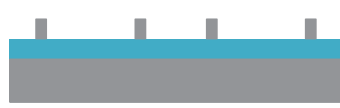

(h) Anisotropic Plasma etching $\mathrm{SiN}$
Fig. 1. Fabrication of silicon nanowires using sidewall technique.

A conformal SiN film with controlled thickness is deposited using PECVD, see Fig. 1(d), followed by anisotropic RIE etching to remove the SiN film on the wafer surface, see Fig. 1(e). Then the $\mathrm{SiO}_{2}$ layer is removed using $\mathrm{HF}$ wet etching, after which the SiN film on the sidewalls of the $\mathrm{SiO}_{2}$ patterns is left, see Fig. 1(f). Next the device layer of the SOI wafer is etched to form SNWs using RIE with the SiN patterns as the 
mask, see Fig. 1(g), followed by RIE etching to remove the SiN mask, see Fig. 1(h).

The SiN masks have a feature size determined by the thickness rather than photolithography. As the thickness can be controlled easily to tens or few hundreds nanometers using normal CMOS technology, it avoids usage of advanced and expensive lithography facilities.

\section{SNW pH Sensors}

Fig. 2 shows the as-fabricated SNW FET. The length of the SNW is $10 \mu \mathrm{m}$. The overall diameter of the nanowire is $350 \mathrm{~nm}$, including a $150 \mathrm{~nm}$ SNW and a $100 \mathrm{~nm}$ silicon oxide insulator. Fig. 3 shows the scheme for flip-chip bonding of SNW with CMOS signal processing circuits, which are fabricated on separate wafers. For $\mathrm{pH}$ measurement, the gate voltage of the SNW FET is applied via a reference electrode immersed into the solution.

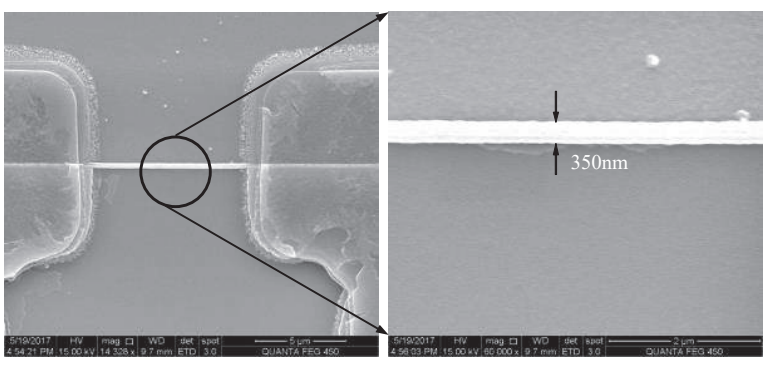

Fig. 2. Silicon nanowire sensor fabricated using sidewall technique.

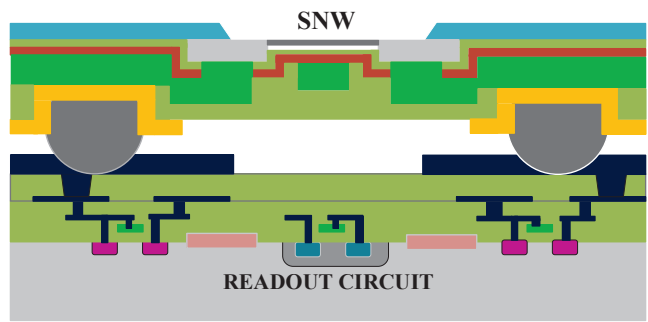

Fig. 3. Flip-chip integration of silicon nanowire with CMOS signal processing circuits.

Fig. 4(a) shows the drain current vs. drain voltages at $\mathrm{pH}$ values from 1 to 12 and constant gate solution voltage $(\mathrm{Vg}=-4 \mathrm{~V})$. The decrease in the absolute value of the threshold voltage causes increase in the drain-source current at a given gate voltage. Fig. 4 (b) shows the current vs. the gate voltage at $\mathrm{pH}$ values from 1 to 12 and constant drain voltage $(\mathrm{Vd}=-3 \mathrm{~V})$. Since the SNW FET is P-type, the proton density at the interface decreases with the $\mathrm{pH}$ values of the gate solution, resulting in increases in the absolute value of the threshold voltage [4].

By using the drain currents, the thresholds to different $\mathrm{pH}$ values can be obtained, as shown in the inset in Fig. 4(b). Using the fitted line, the sensitivity of the SNW FET to $\mathrm{pH}$ values are calculated as $54.5 \mathrm{mV} / \mathrm{pH}$. This sensitivity is slightly lower than the theoretical Nernstian value $(59.5 \mathrm{mV} / \mathrm{pH}, 300 \mathrm{~K})$ [5], indicating that the SNW FET pH sensors fabricated using sidewall mask technology is highly sensitive.
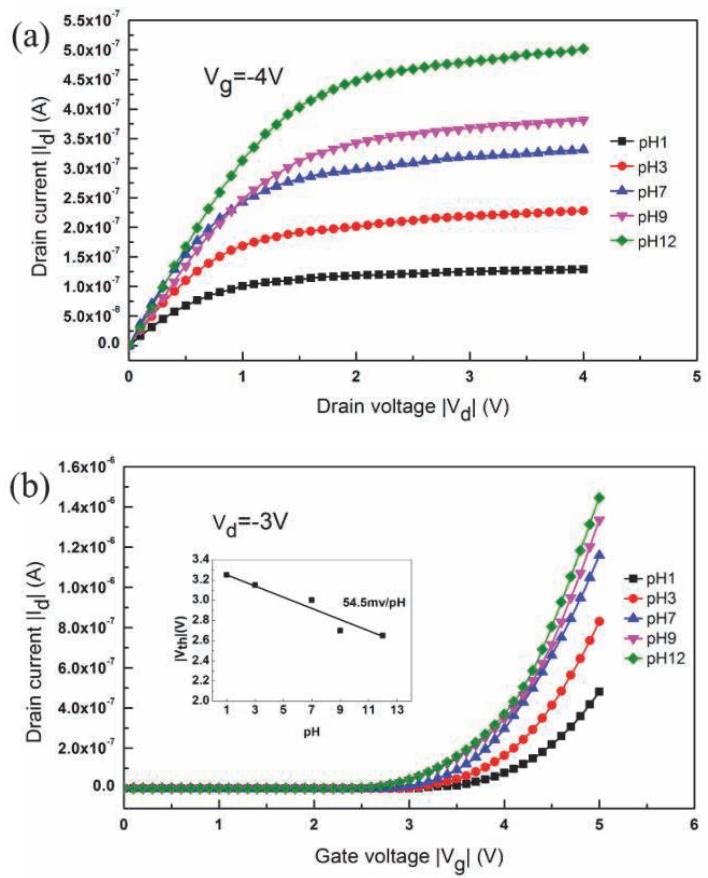

Fig. 4. The responses of the SNW FET vs different. $\mathrm{pH}$ values. (a) Drain current vs. drain voltages. (b) The current vs. gate voltage.

\section{Conclusion}

A method for fabrication of silicon nanowires has been demonstrated. It can obtain tens or few hundreds nanometer feature sizes readily using the thickness of a thin film rather than advanced lithography facilities. This allows fabrication of SNWs with high output, low cost, and easy availability. The SNW $\mathrm{pH}$ sensor shows a high sensitivity of $54.5 \mathrm{mV} / \mathrm{pH}$, close to the theoretical Nernstian limit of $59.5 \mathrm{mV} / \mathrm{pH}$.

\section{References}

[1] K. Chen, et al., Silicon nanowire field-effect transistor-based biosensors for biomedical diagnosis and cellular recording investigation, Nano today, 6, 131-154, 2011.

[2] R. M. Penner, Chemical sensing with nanowires, Annu. Rev. Anal. Chem., 5, 461-485, 2012.

[3] P. W. Wachulak, et al., Patterning of nano-scale arrays by table-top extreme ultraviolet laser interferometric lithography, Optics Express, 15, 3465-3469, 2007.

[4] H.-J. Jang and W.-J. Cho, Fabrication of highperformance fully depleted silicon-on-insulator based dual-gate ion-sensitive field-effect transistor beyond the Nernstian limit, Appl. Phys. Lett., 100, 073701, 2012.

[5] J.-H. Ahn, et al., A pH sensor with a double-gate silicon nanowire field-effect transistor, Appl. Phys. Lett., 083701, 2013. 